\title{
PATH FOLLOWING PROBLEM FOR UAV CARRYING PENDULUM
}

\author{
A. S. SHAHINYAN
}

Chair of Mechanics, YSU, Armenia

The linearized dynamics of a UAV is considered along with a pendulum hanging from it. The state trajectories of the center of mass of the UAV are given. Given the trajectory of the center of mass of the UAV and the state trajectory of its yaw angle, we have to find the control actions and conditions under which the UAV would follow the path while holding the pendulum stable around its lower equilibrium point. The problem is solved using the method for solving inverse problems of dynamics. All the state trajectories of the system and all the control actions are calculated. The condition is obtained under which a solution to the path following problem exists. A specified simple trajectory is chosen as an example for visualizing the results.

\section{https://doi.org/10.46991/PYSU:A/2021.55.1.056}

Keywords: dynamic systems, control theory, optimal stabilization, quadcopter.

Introduction. Control problems of UAVs have important applications in both science and life. The history of UAVs, the examination and the research about the UAVs are thoroughly discussed in [1]. The trajectory tracking and path following problems of UAVs are extremely relevant. The fact is that such kind of problems are considered and discussed by a huge number of researchers recently and nowadays. For an overview of articles in this area, see the survey paper [2].

In this article, the dynamics of a UAV is considered along with a pendulum hanging from it. The older article [3] is used to represent the linearized dynamics of the UAV-pendulum system. Then, the state trajectories of the center of mass of the UAV are given. The problem is to calculate the control actions and conditions under which the UAV will follow the path, keeping the pendulum in a stable state around the equilibrium point.

Using the method for solving inverse problems of dynamics, all the state trajectories of the system and all the control actions are calculated. The results we gained, that is, the control inputs and state trajectories, are given both in analytic form and shown in the form of graphs that were generated from virtual simulations for a specified simple trajectory example.

\footnotetext{
* E-mail: a.s.shahinyan@gmail.com
} 
Modelling of the System. To derive the purely theoretical dynamics of a UAV, we fix the coordinate system $O x y z$. Let $O$ be the origin. We also need another coordinate system $O_{B} x_{B} y_{B} z_{B}$ fixed at the center of mass $O_{B}$ of the UAV (Fig. 1). The torques and forces generated by each of the propellers are numbered 1 to 4 [3].

Let $\xi=\left(\begin{array}{lll}x & y & z\end{array}\right)^{T}$ be the coordinates of the center of mass of the UAV relative to the $O x y z$ system. By definition, the center of mass of the UAV coincides with the origin of the $O_{B} x_{B} y_{B} z_{B}$ coordinate system. Let us describe the inclined position of the UAV about the point $O_{B}$ using yaw $(\Psi)$, pitch $(\Phi)$ and roll $(\Theta)$ angles. Then we will have two vectors describing the position of the UAV:

$$
\xi=\left(\begin{array}{lll}
x & y & z
\end{array}\right)^{T}, \eta=(\Phi \Theta \Psi)^{T},
$$

as well as the linear $\bar{V}_{B}$ and angular $\bar{v}$ velocities:

$$
\bar{V}_{B}=\left(\begin{array}{lll}
\bar{V}_{B x} & \bar{V}_{B y} & \bar{V}_{B}
\end{array}\right)^{T}, \bar{v}=\left(\begin{array}{lll}
p & q & r
\end{array}\right)^{T} .
$$

In this notation, the dynamics of the system is described by the following system $[3,4]$ :

$$
\left\{\begin{array}{l}
\ddot{x}=\frac{T}{M} C_{\Psi} C_{\Theta} C_{\Phi}+\frac{T}{M} S_{\Psi} S_{\Theta} \\
\ddot{y}=\frac{T}{M} S_{\Psi} S_{\Theta} C_{\Phi}+\frac{T}{M} C_{\Psi} S_{\Theta} \\
\ddot{z}=-g+\frac{T}{M} C_{\Theta} C_{\Phi} \\
\dot{\Phi}=p+\frac{S_{\Phi} S_{\Theta}}{C_{\Theta}} q+\frac{C_{\Phi} S_{\Theta}}{C_{\Theta}} r \\
\dot{\Theta}=C_{\Phi} q-S_{\Phi} r \\
\dot{\Psi}=\frac{S_{\Phi}}{C_{\Theta}+\frac{C_{\Phi}}{C_{\Theta}} r} \\
\dot{p}=\frac{\left(I_{y y}-I_{z z}\right) q r}{I_{x x}}-I_{r} \frac{q}{I_{x x}} \omega_{\Gamma}+\frac{\tau_{\Phi}}{I_{x x}} \\
\dot{q}=\frac{\left(I_{z z}-I_{x x}\right) p r}{I_{y y}}-I_{r} \frac{p}{I_{y y}} \omega_{\Gamma}+\frac{\tau_{\Theta}}{I_{y y}} \\
\dot{p}=\frac{\left(I_{x x}-I_{y y}\right) p q}{I_{z z}}-I_{r} \frac{r}{I_{z z}} \omega_{\Gamma}+\frac{\tau_{\Psi}}{I_{z z}}
\end{array}\right.
$$

Here the following notations are adopted:

$$
\begin{aligned}
& C_{\alpha}=\cos \alpha, S_{\alpha}=\sin \alpha, M=m_{U A V}+m_{P}, \\
& \tau_{B}=\left(\begin{array}{c}
\tau_{\Phi} \\
\tau_{\ominus} \\
\tau_{\Psi}
\end{array}\right)=\left(\begin{array}{c}
l k\left(-\omega_{2}^{2}+\omega_{4}^{2}\right) \\
l k\left(-\omega_{1}^{2}+\omega_{3}^{2}\right) \\
\sum_{i} \tau_{i}
\end{array}\right), \\
& T=\sum_{i} F_{i}=\sum_{i} k \omega_{i}^{2}, \bar{T}=\left(\begin{array}{lll}
0 & 0 & T
\end{array}\right)^{T} .
\end{aligned}
$$



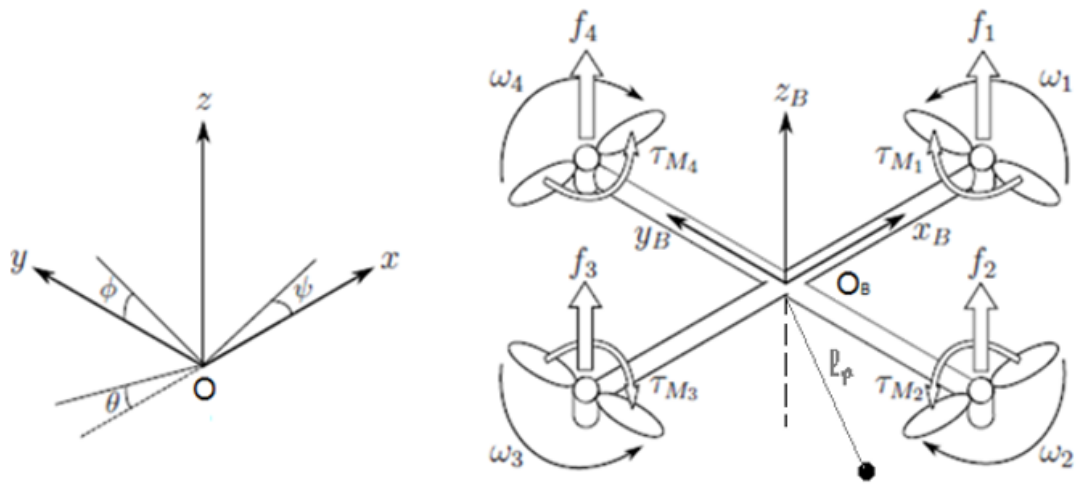

Fig. 1.

As for the mathematical model of the pendulum, we will consider its dynamics in the coordinate system $O_{B} x_{B} y_{B} z_{B}$. So, the dynamics of the pendulum will be as follows [4]:

$$
\left\{\begin{aligned}
\ddot{x}_{p}= & \frac{1}{\left(l_{p}^{2}+y_{p}^{2}\right) \zeta^{2}}\left(-x_{p}^{4} \ddot{x}_{p}-\left(l_{p}^{2}-y_{p}^{2}\right) \ddot{x}_{p}-2 x_{p}^{2}\left(y_{p} \dot{x}_{p} \dot{y}_{p}-\left(l_{p}^{2}-y_{p}^{2}\right) \ddot{x}_{p}\right)\right. \\
& +x_{p}^{3}\left(\dot{y}_{p}^{2}+y_{p} \ddot{y}_{p}+\zeta(g+\ddot{z})\right)+x_{p}\left(-l_{p}^{2} y_{p} \ddot{y}_{p}+y_{p}^{3} \ddot{y}_{p}+y_{p}^{2}\left(\dot{x}_{p}^{2}+\zeta(g+\ddot{z})\right)\right. \\
& \left.\left.+l_{p}^{2}\left(-\dot{x}_{p}^{2}-\dot{y}_{p}^{2}-\zeta(g+\ddot{z})\right)\right)\right) \\
\ddot{y}_{p}= & \frac{1}{\left(l_{p}^{2}+x_{p}^{2}\right) \zeta^{2}}\left(-y_{p}^{4} \ddot{y}_{p}-\left(l_{p}^{2}-x_{p}^{2}\right) \ddot{x}_{p}-2 y_{p}^{2}\left(x_{p} \dot{x}_{p} \dot{y}_{p}-\left(l_{p}^{2}-x_{p}^{2}\right) \ddot{y}_{p}\right)\right. \\
& +y_{p}^{3}\left(\dot{x}_{p}^{2}+x_{p} \ddot{x}_{p}+\zeta(g+\ddot{z})\right)+y_{p}\left(-l_{p}^{2} x_{p} \ddot{x}_{p}+x_{p}^{3} \ddot{x}_{p}+x_{p}^{2}\left(\dot{y}_{p}^{2}+\zeta(g+\ddot{z})\right)\right. \\
& \left.\left.+l_{p}^{2}\left(-\dot{x}_{p}^{2}-\dot{y}_{p}^{2}-\zeta(g+\ddot{z})\right)\right)\right),
\end{aligned}\right.
$$

where $l_{p}$ denotes the length of the pendulum. Using the formula for the center of mass of a system

$$
\bar{r}_{c}=\frac{m_{1} \bar{r}_{1}+m_{2} \bar{r}_{2}}{m_{1}+m_{2}},
$$

where $\bar{r}_{c}=\left(\begin{array}{lll}x_{c} & y_{c} & z_{c}\end{array}\right)^{T}, \bar{r}_{1}=\bar{\xi}=\left(\begin{array}{lll}x & y & z\end{array}\right)^{T}$, and $\bar{r}_{2}=\bar{r}_{p}=\left(\begin{array}{lll}x+x_{p} & y+y_{p} & z+z_{p}\end{array}\right)^{T}$, and linearizing the dynamics around the origin of the fixed coordinate system, we get the following linear dynamics:

$$
\begin{aligned}
& \dot{x}_{1}=x_{2}, \dot{x}_{2}=\frac{g}{4} x_{8}-\frac{g}{2 l_{p}} x_{13}, \dot{x}_{3}=x_{4}, \dot{x}_{4}=-\frac{g}{4} x_{7}-\frac{g}{2 l_{p}} x_{15}, \dot{x}_{5}=x_{6}, \dot{x}_{6}=u_{1}, \\
& \dot{x}_{7}=x_{10}, \dot{x}_{8}=x_{11}, \dot{x}_{9}=x_{12}, \dot{x}_{10}=u_{2}-\frac{g}{I_{x x}} x_{1} 5, \dot{x}_{11}=u_{3}-\frac{g}{I_{y y}} x_{13}, \dot{x}_{12}=\frac{u_{4}}{I_{z z}}, \\
& \dot{x}_{13}=x_{14}, \dot{x}_{14}=-\frac{g}{2} x_{8}-\frac{g}{l_{p}} x_{13}, \dot{x}_{15}=x_{16}, \dot{x}_{16}=\frac{g}{2} x_{7}-\frac{g}{l_{p}} x_{15},
\end{aligned}
$$

where $u_{1}=\frac{T}{M}-g, u_{2}=\tau_{\Phi}, u_{3}=\tau_{\ominus}, u_{4}=\tau_{\Psi}, m_{U A V}=m_{p}$ and the following notations are introduced:

$$
\begin{aligned}
& x_{1}=x_{c}, x_{2}=\dot{x}_{c}, x_{3}=y_{c}, x_{4}=\dot{y}_{c}, x_{5}=z_{c}, x_{6}=\dot{z}_{c}, x_{7}=\Phi, x_{8}=\Theta, \\
& x_{9}=\Psi, x_{10}=p, x_{11}=q, x_{12}=r, x_{13}=x_{p}, x_{14}=\dot{x}_{p}, x_{15}=y_{p}, x_{16}=\dot{y}_{p} .
\end{aligned}
$$


Using Kalman's rule, one can check that the system (6) is fully controllable. So, now we are at a point where we can define the problem and we can go ahead to show how we solved it.

Problem Definition. Given the system (6) and the trajectories of its center of mass, we have to calculate the control actions $u_{i}(i=1, \ldots, 4)$ and the conditions under which the UAV will follow the path, keeping the pendulum stable around its lower equilibrium point.

In other words, we are given the trajectory of the center of mass of the UAV and the state trajectory of its yaw angle, and we are required to find the control actions $u_{i}(i=1, \ldots, 4)$ and the conditions under which the UAV will fly along the given path and at the same time maintain the stability of the pendulum at the equilibrium point.

Solution. Suppose the trajectory of the center of mass of the UAV is given as

$$
x_{1}=g_{1}(t), x_{3}=g_{3}(t) x_{5}=g_{5}(t),
$$

and the yaw angle state trajectory is

$$
x_{9}=g_{9}(t),
$$

where $g_{1}(t), g_{3}(t) \in C^{4}[0 ; \infty), g_{5}(t), g_{9}(t) \in C^{2}[0, \infty)$. Then from the first, third and fifth equations of the system (6) we will have

$$
x_{2}=g_{1}^{\prime}(t), x_{4}=g_{3}^{\prime}(t), x_{6}=g_{5}^{\prime}(t) .
$$

$x_{13}$ and $x_{15}$ can be calculated from the second and fourth equations of the system (6). Hence, we can also get $x_{14}$ and $x_{16}$ :

$$
\begin{aligned}
& x_{13}=x_{13}(0) \cos (k t)+\frac{x_{14}(0)}{k} \sin (k t)-\frac{2}{k} \int_{0}^{t} \sin (t-\tau) g_{1}^{\prime \prime}(\tau) d \tau, \\
& x_{15}=x_{15}(0) \cos (k t)+\frac{x_{16}(0)}{k} \sin (k t)-\frac{2}{k} \int_{0}^{t} \sin (t-\tau) g_{3}^{\prime \prime}(\tau) d \tau, \\
& x_{14}=-x_{13}(0) k \sin (k t)+x_{14} \cos (k t)-2 \int_{0}^{t} \cos (t-\tau) g_{1}^{\prime \prime}(\tau) d \tau, \\
& x_{16}=-x_{15}(0) k \sin (k t)+x_{16} \cos (k t)-2 \int_{0}^{t} \cos (t-\tau) g_{3}^{\prime \prime}(\tau) d \tau,
\end{aligned}
$$

where $k^{2}=\frac{2 g}{l_{p}}>0$. State trajectories of $x_{7}=\Phi$ pitch and $x_{8}=\Theta$ roll angles can be gained from the sixteenth and fourteenth equations of the system (6), respectively. We will have:

$$
\begin{gathered}
x_{7}=-\frac{4}{g} g_{3}^{\prime \prime}(t)-\frac{2}{l_{p}} x_{15}(t), \\
x_{8}=\frac{4}{g} g_{1}^{\prime \prime}(t)+\frac{2}{l_{p}} x_{13}(t) .
\end{gathered}
$$


Using the seventh and eighth equations of the system (6), we can acquire $x_{10}$ and $x_{11}$ :

$$
\begin{aligned}
& x_{10}=\frac{4}{g} g_{3}^{\prime \prime \prime}(t)-\frac{2}{l_{p}} x_{15}^{\prime}(t), \\
& x_{11}=\frac{4}{g} g_{1}^{\prime \prime \prime}(t)+\frac{2}{l_{p}} x_{13}^{\prime}(t) .
\end{aligned}
$$

It only remains to get $x_{6}$ and $x_{12}$, for which we will have:

$$
\begin{aligned}
& x_{6}=g_{5}^{\prime}(t), \\
& x_{12}=g_{9}^{\prime}(t) .
\end{aligned}
$$

Now that we have all the state trajectories of the system (6), we can calculate the control actions $u_{i}(i=1, \ldots 4)$. Using Eq. (6) and the Eqs. (8)-(20) we will have:

$$
\left\{\begin{array}{l}
u_{1}=\dot{x}_{6}=g_{5}^{\prime \prime}(t) \\
u_{2}=\dot{x}_{10}+\frac{g}{I_{x x}} x_{15}=-\frac{4}{g} g_{3}^{I V}(t)-\frac{2}{l_{p}} \ddot{x}_{15}+\frac{g}{I_{x x}} x_{15}(t) \\
u_{3}=\dot{x}_{11}+\frac{g}{I_{y y}} x_{13}=\frac{4}{g} g_{1}^{I V}(t)-\frac{2}{l_{p}} \ddot{x}_{13}+\frac{g}{I_{y y}} x_{13}(t) \\
u_{4}=\dot{x}_{12}=g_{9}^{\prime}(t) .
\end{array}\right.
$$

Suppose that the pendulum is allowed to move no more than $R(R>0)$ from its equilibrium point. This means that states of the pendulum $x_{p}$ and $y_{p}$ must satisfy the following inequality:

$$
x_{p}^{2}+y_{p}^{2} \leq R^{2} .
$$

So, in order to ensure that the UAV follows the path given by the functions (8), we will need to construct the control actions as given in (21).

Numerical Example. To demonstrate the results visually, let's carry out the above calculations for a simple case scenario. So, in our example, we will consider the following path-defining functions:

$$
\left\{\begin{array}{l}
x_{1}(t)=g_{1}(t)=r \sin (\omega t) \\
x_{3}(t)=g_{3}(t)=r \cos (\omega t) \\
x_{5}(t)=g_{5}(t)=v t \\
x_{9}(t)=0
\end{array}\right.
$$

where $r=5 \mathrm{~m}, v=0.1 \mathrm{~m} / \mathrm{s}, \omega=0.5 \mathrm{~s}^{-1}$. As for the other parameters of the system, we take $g=9.8 \mathrm{~m} / \mathrm{s}^{2}, I_{x x}=I_{y y}=0.004856 \mathrm{~kg} \cdot \mathrm{m}^{2}, I_{z z}=0.008801 \mathrm{~kg} \cdot \mathrm{m}^{2}$. By following the steps outlined in the solution section, we will have our integrated 
system as follows:

$$
\left\{\begin{array}{l}
x_{1}(t)=5 \sin (0.5 t) \\
x_{2}(t)=2.5 \cos (0.5 t) \\
x_{3}(t)=5 \cos (0.5 t) \\
x_{4}(t)=-2.5 \sin (0.5 t) \\
x_{5}(t)=0.1 t \\
x_{6}(t)=0.1 \\
x_{7}(t)=0.51 \cos (0.5 t)+2(0.45(-0.29 \cos (0.5 t)+0.29 \cos (4.43 t))) \\
x_{8}(t)=-0.51 \sin (0.5 t)-2(0.45(-0.29 \sin (0.5 t)+0.03 \sin (4.43 t))) \\
x_{9}(t)=0 \\
x_{10}(t)=-0.26 \sin (0.5 t)+0.9(0.14 \sin (0.5 t)-1.27 \sin (4.43 t)) \\
x_{11}(t)=-0.26 \cos (0.5 t)+0.9(0.14 \cos (0.5 t)-0.14 \cos (4.43 t)) \\
x_{12}(t)=0 \\
x_{13}(t)=0.45(0.29 \sin (0.5 t)-0.03 \sin (4.43 t)) \\
x_{14}(t)=2(0.03 \cos (0.5 t)-0.03 \cos (4.43 t)) \\
x_{15}(t)=-0.45(-0.29 \cos (0.5 t)+0.29 \cos (4.43 t)) \\
x_{16}(t)=-2(0.03 \sin (0.5 t)-0.29 \sin (4.43 t)) .
\end{array}\right.
$$

As for the control actions, we will have:

$$
\left\{\begin{aligned}
u_{1}(t) & =0 \\
u_{2}(t) & =-0.13 \cos (0.5 t)+2018.12(0.45(0.29 \cos (0.5 t)-0.29 \cos (4.43 t))) \\
& +0.9(0.07 \cos (0.5 t)-5.6 \cos (4.43 t)) \\
u_{e}(t) & =0.13 \sin (0.5 t)-2018.12(0.45(0.29 \sin (0.5 t)-0.03 \sin (4.43 t))) \\
& +0.9(0.07 \sin (0.5 t)-0.63 \sin (4.43 t)) \\
u_{4}(t) & =0 .
\end{aligned}\right.
$$

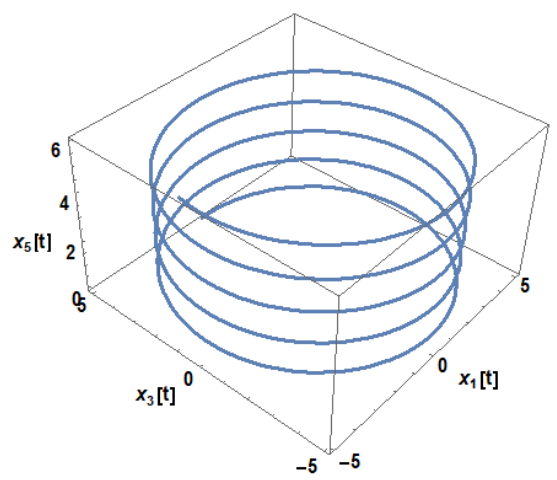

Fig. 2. Trajectory of center of mass of the UAV.

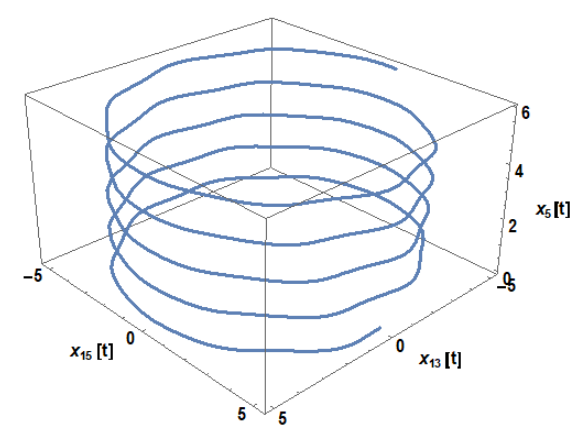

Fig. 3. Trajectory of the pendulum. 
So, the maximum deviation of the pendulum from its equilibrium position can be calculated, and it is equal to $R=0.26 \mathrm{~m}$, which in this case equals to approximately $5.2 \%$ of the trajectory radius.

And finally, the results of the above calculations are demonstrated in the form of graphs (Figs. 2, 3, 4).

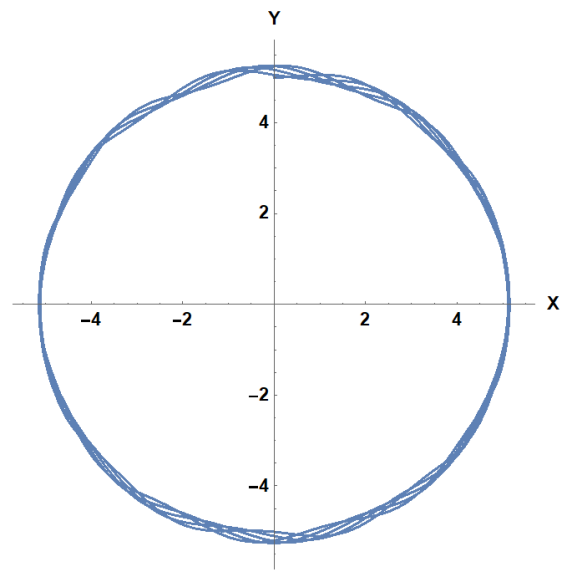

Fig. 4. The projection of the absolute motion of the pendulum on the Oxy plane.

Received 15.03.2021

Reviewed 29.03.2021

Accepted 12.04.2021

\section{R E F E R E N C E S}

1. Shahinyan A.S. Hybrid Control of Motion of an Unmanned Aerial Vehicle, Carrying an Inverted Pendulum. Proc. NAS RA. Mechanics 73 : 2 (2020), 69-78.

2. Rubi B., Perez R., Morcego B. A Survey of Path Following Control Strategies for UAVs Focused on Quadrotors. J. Intell. Robot. Syst. 98 (2020), 241-265. http://doi .org/10.1007/s10846-019-01085-z

3. Luukkonen T. Modelling and Control of Quadcopter. School of Science. Mat-2.4108, Independent Research Project in Applied Mathematics. Espoo, 22.09.2011, 26 p.

4. Buchholz N.N. The Main Course of Theoretical Mechanics. V. 2. Moscow, Nauka (1972), 332 p. (in Russian). 


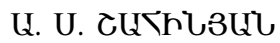

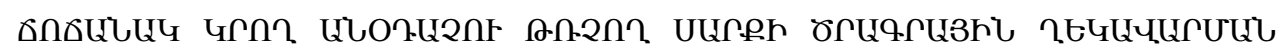
Uh bul?he

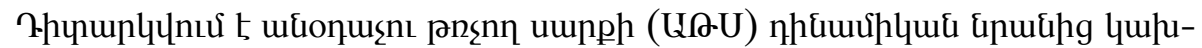

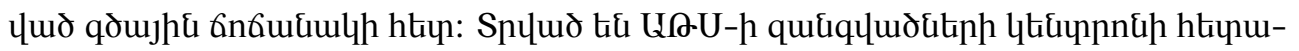

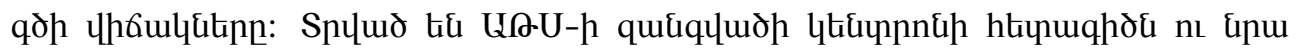

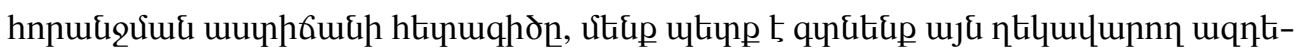

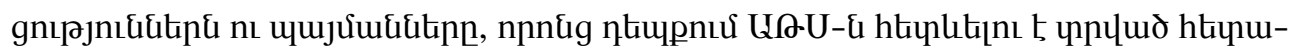

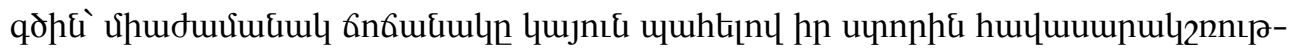

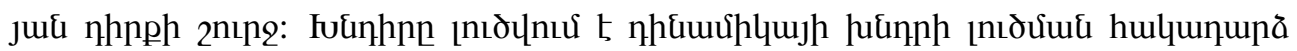

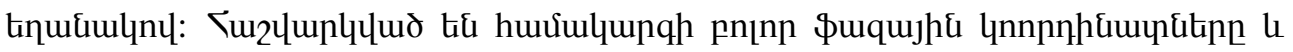

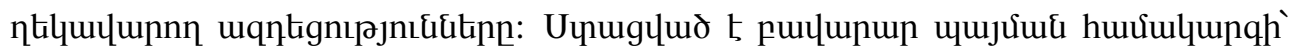

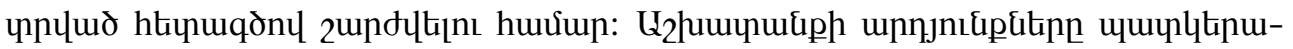

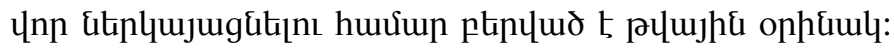

\section{А. С. ШАГИНЯН}

\section{ЗАДАЧА СЛЕДОВАНИЯ ПО ТРАЕКТОРИИ БПЛА, НЕСУЩЕГО МАЯТНИК}

Рассмотрена линеаризованная динамика беспилотного летательного аппарата (БПЛА) с маятником, свисающим с него. Приведены состояния траектории центра масс БПЛА. Имея траекторию центра масс БПЛА и траекторию состояния угла рыскания, мы должны найти управляющие воздействия и условия, при которых БПЛА будет следовать по траектории, удерживая маятник в устойчивом состоянии вокруг его нижней точки равновесия. Задача решается методом обратного решения задачи динамики. Рассчитаны все траектории состояний системы и все управляющие воздействия. Получено условие, при котором существует решение задачи о пути следования. Заданная простая траектория выбрана в качестве примера для визуализации результатов. 